\title{
NEUTRON DEPOLARISATION IN COCr LAYERS TO STUDY THE PERPENDICULAR MAGNETIC DOMAIN STRUCTURE
}

\author{
W.H. KRAAN and M.Th. REKVELDT \\ Interuniversitair Reactor Insituut, $2629 \mathrm{JB}$ Delft, The Netherlands \\ K. HEMMES and J.C. LODDER \\ Twente University of Technology, P.O. Box 217, 7500 AE Enschede, The Netherlands
}

\begin{abstract}
Polarised neutrons $(\lambda=0.47 \mathrm{~nm})$ are transmitted through sputtered $\mathrm{CoCr}$ films. From the measured diagonal elements of the depolarisation matrix it appears that the effective thickness $h_{\text {eff }}$ of the films is about $50 \%$ of the actual thickness. When a soft magnetic NiFe film is deposited before or after deposition of the $\mathrm{CoCr}, h_{\mathrm{eff}}$ is 67 and $60 \%$ of the actual thickness, respectively, due to the disappearance of the flux closure in the $\mathrm{CoCr}$ film. From the angular dependence of the diagonal elements compared with computer simlation the "chain of column" model is supported. Our results indicate that magnetic flux is closed by "branched" spikes at least on the substrate side of the $\mathrm{CoCr}$ film.
\end{abstract}

\section{Introduction}

Sputtered $\mathrm{CoCr}$ layers consisting of conically shaped crystals (columns) with the hcp c-axis perpendicular to the film plane are at present widely studied because of their unique properties for using them as a perpendicular recording medium with high density [1]. The morphology and crystal structure and therefore the magnetic properties of these films depend strongly on the deposition and growth conditions. In order to understand the magnetisation process, domain studies are of great importance. In particular, the connection between the domain and column structure of these films is by no means clear.

The domain dimensions predicted in both "particle" models and "continuous" models [2] for such films are too small to have been observed up to now by Kerr microscopy or by the Bitter technique. Therefore, we have transmitted polarised neutrons $(\lambda=0.47 \mathrm{~nm})$ through these films and measured the diagonal elements $D_{x x}, D_{y y}$ and $D_{z z}$ of the depolarisation matrix [3]. The average Larmor precession of the polarisation vector in the spontaneous magnetisation $\boldsymbol{M}_{\mathrm{s}}$ inside the domains is a measure for the mean domain size in the transmission direction.

If the domains extend through the thickness (or

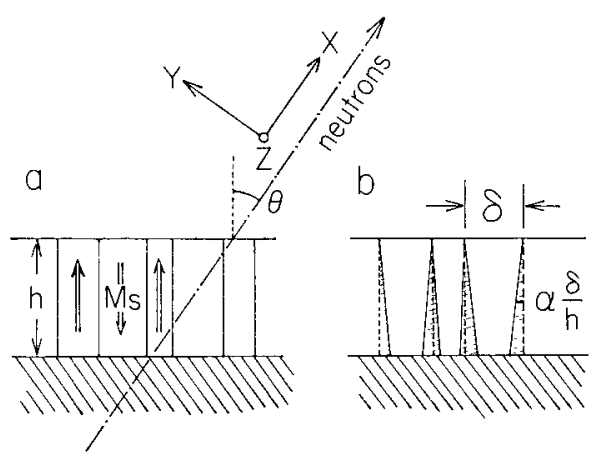

Fig. 1. (a) Domain model of a cross section of the film with domains extending through the thickness $(h)$. (b) Domain model with (shaded) areas between oblique domain walls where no effective Larmor precession occurs.

"height") $h$ of the film (fig. 1a) and when the neutrons pass perpendicularly, the precession angle is

$\phi=5.72 \times 10^{8} \times\left(M_{\mathrm{s}} h\right) \lambda$,

where $M_{\mathrm{s}}$ is in $\mathrm{A} / \mathrm{m}$ and $\lambda$ and $h$ are in meters. For CoCr films with $h=1 \mu \mathrm{m}$ and $M_{\mathrm{s}}=460 \mathrm{kA} / \mathrm{m}$ one finds $\phi \approx 5^{\circ}$, hence $D_{z z}=\cos \phi \approx 0.996$. This means that transmission through a package of 14 films should give a good measurable effect: $D_{z z}=$ $\cos ^{14} \phi \approx 0.95$. 


\section{Observations and interpretation}

The upper part of table I which was published earlier [4] contains data from films of 4 thicknesses. The values for $\phi$ and $\cos ^{14} \phi$ are obained from $\left(M_{\mathrm{s}} L\right)$ using eq. (1). Dividing $\phi_{\text {eff }}=\cos ^{-1}$ $\left[\left(D_{z z}\right)^{1 / 14}\right]$ by $\phi$ yields $h_{\text {eff }} / h$. It follows that $h_{\text {eff }}$ is about half the film thickness, with a tendency to increase for thinner films. This is partly due to flux closure at the domain ends which "consumes" part of the height of the film; furthermore, due to the fact that the domain walls are not exactly perpendicular to the plane of the film and due to the different morphology of the so-called "initial layer".

To investigate the flux closure, we have sputtered a soft magnetic $0.05 \mu \mathrm{m}$ thick $\mathrm{Ni}_{81} \mathrm{Fe}_{19}$ film on top of sample \#3 in order to short circuit part of the magnetic flux of the $\mathrm{CoCr}$ domains outside the film. Moreover, we have prepared sample \#5 consisting of $\mathrm{CoCr}$ on top of $\mathrm{NiFe}$. It appears that $h_{\text {eff }}$ is significantly bigger than in untreated $\mathrm{CoCr}$ films. However, we note that the morphology of sample \#5 is quite different from the others.

In non-perpendicular transmission, fig. 2 gives the result for $D_{x x}$ and $D_{z z}$ in sample \#1 as a function of $\theta$. The average domain section length $\langle\delta\rangle$ of the film may be inferred from the result for $D_{z z}$ by comparison with a computer simulation using the model of fig. 1a. As parameters we take:

(i) the thickness $h$ of the film (taken equal to $h_{\text {eff }}$;

(ii) the average $\langle\delta\rangle$ as seen in a fixed direction in the plane of the film;

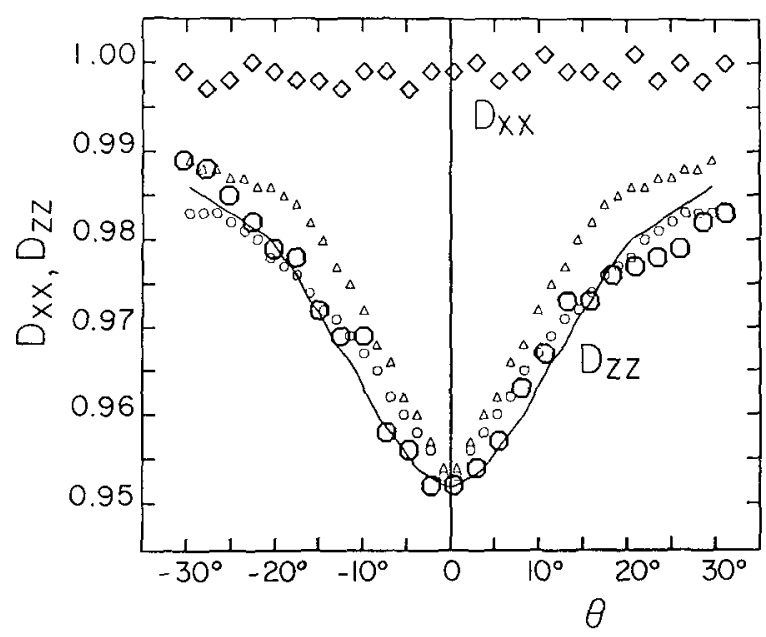

Fig. 2. Experimental results of sample \#1 for $D_{x x}$ and $D_{z z}$ (big symbols) and simulated results for $D_{z z}$ (small symbols) with the model of fig. 1a and with $h=h_{\text {eff }}$. The circles and triangles correspond to the domain distributions of fig. $3 a$ and $3 b$, respectively. The solid line corresponds to the model in fig. $1 \mathrm{~b}$ with the random $\delta$-distribution with $\alpha=0.2$ and with a height of $0.8 h$.

(iii) the distribution of lengths $\delta$.

The average $\langle\delta\rangle$ need not be equal to the column diameter. The simulation has been carried out according to a procedure described earlier [5]. The distribution of $\delta$ has been found from a photograph of sample \#1 made with a TEM replica technique. In a graph of the crystal boundaries derived from this photograph we have assigned + or - magnetisation at random (fig. 3a) to individual crystals and measured the intersections along a set of parallel lines through the magnetic domains consisting of crystals of equal

Table I

Comparison of measured $D_{z z}$ with expected $\cos ^{14} \phi$, expressed in $h_{\mathrm{eff}} / h$ for various samples

\begin{tabular}{llllllll}
\hline $\begin{array}{l}\text { Sample } \\
\text { \#) }\end{array}$ & $\begin{array}{l}h_{\text {NiFe }} \\
(\text { bottom) } \\
(\mu \mathrm{m})\end{array}$ & $\begin{array}{l}h_{\mathrm{CoCr}} \\
(\mu \mathrm{m})\end{array}$ & $\begin{array}{l}h_{\mathrm{NiFe}} \\
(\mathrm{top}) \\
(\mu \mathrm{m})\end{array}$ & $\begin{array}{l}\left(M_{\mathrm{s}} h\right)_{\mathrm{CoCr}} \\
(\mathrm{A})\end{array}$ & $\cos ^{14} \phi$ & $D_{z z}$ & $h_{\mathrm{eff}} / h$ \\
\hline 1 & - & 0.95 & - & 0.421 & 0.913 & $0.952 \pm 0.003$ & $0.72 \pm 0.04^{\mathrm{a})}$ \\
2 & - & 1.90 & - & 0.855 & 0.686 & $0.88 \pm 0.005$ & $0.58 \pm 0.03$ \\
3 & - & 3.81 & - & 1.737 & 0.202 & $0.67 \pm 0.01$ & $0.51 \pm 0.02$ \\
4 & - & 4.76 & - & 2.185 & 0.075 & $0.57 \pm 0.01$ & $0.48 \pm 0.02$ \\
3 & - & 3.8 & 0.06 & 1.74 & 0.202 & $0.50 \pm 0.01$ & $0.61 \pm 0.02$ \\
5 & 0.04 & 4.2 & - & 1.94 & 0.138 & $0.42 \pm 0.01$ & $0.67 \pm 0.02$ \\
\hline
\end{tabular}

a) In ref. 4 we reported $0.61 \pm 0.07$. The present figure is obtained after more accurate experiments. 


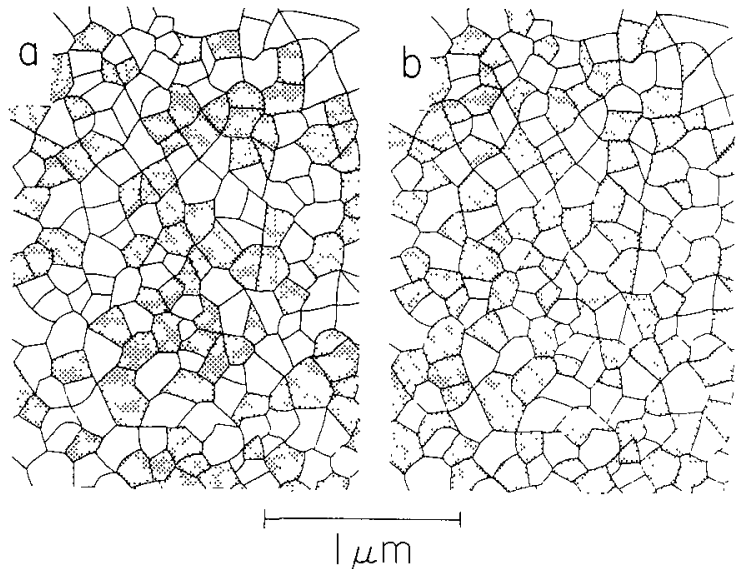

Fig. 3. Graph of the crystal boundaries in sample \#1 obtained from a TEM replica photograph with + or - magnetisation assigned (a) at random and (b) with maximum number of neighbouring crystals with anti-parallel magnetisation.

sign. This leads to a distribution with a tail and $\langle\delta\rangle=0.218 \mu \mathrm{m}$. Maximizing the numbers of pairs of neighbouring crystals with opposite sign (i.e. minimizing magnetostatic energy) gives fig. 3b with $\langle\delta\rangle=0.163 \mu \mathrm{m}$. Both represent a "chain of column" model. The outcome of the simulations is plotted in fig. 2. It appears that the "random" choice agrees best with the depolarisation results.

\section{Discussion and conclusion}

Comparing the measurements with the simulations we conclude that the $\langle\delta\rangle$ value obtained with the random $\delta$ distribution is correct within $10 \%$. We have derived that the average thickness of a layer of closure domains with in-plane magnetisation is about $\frac{1}{4}\langle\delta\rangle$, so in sample \#1 about $0.05 \mu \mathrm{m}$. This gives a reduction of $h_{\mathrm{eff}}$ by $0.1 \mu \mathrm{m}$, hence $h_{\text {eff }} / h$ should be $0.8-0.9$. Such domains cannot fully account for the measured $h_{\mathrm{eff}} / h$ (see table I). It can be derived that the increase in $h_{\text {eff }} / h$ after supplying a NiFe layer is about 2 times $\frac{1}{4}\langle\delta\rangle$. This is also found in the experiment after applying the top NiFe layer (table I, sample \#3). The experiment on sample \#5 with $\mathrm{NiFe}$ on the substrate side preliminarily indicates that $h_{\mathrm{eff}} / h$ is higher than for films with $\mathrm{NiFe}$ on top.

As an alternative flux closure structure we consider a structure consisting of "spikes" of opposite sign grown from the magnetically charged ends of each domain. Such a structure in effect provides a short circuit of magnetic flux within the height of the film by the formation of higher order spikes ("branching") [6,7]. It may be shown that $h_{\text {eff }}$ can be much lower than in the first model. To evaluate the effect of such spikes we have repeated the simulations with the random $\delta$ distribution using the model of fig. 1b. On both sides of each domain a wedge-shaped area with a top angle $\alpha \cdot \delta / h$ is shaded in fig. 1b. In these areas no Larmor precession occurs. The best fit with the experimental data of sample \#1 is obtained with $h_{\text {eff }} / h=0.8$ and $\alpha=0.2$ (solid line in fig. 2).

In summary we can state that the neutron depolarisation technique measures the domain size perpendicular to the plane in $\mathrm{CoCr}$ films with an accuracy in the thinnest films $(\sim 1 \mu \mathrm{m})$ up to $0.02 \mu \mathrm{m}$. The average domain section length $\langle\delta\rangle$ determined by the "chain of column" model in fig. 3 fits quite well with results obtained by the neutron depolarisation experiments giving the same accuracy. Our observations give support for a model by Privorotskii [6] in which magnetic flux is closed by so-called "branching" spikes, at least on the substrate side of the film.

\section{References}

[1] S. Iwasaki, IEEE Trans. Magn. MAG-16 (1980) 71.

[2] T. Wielinga, J.C. Lodder and J. Worst, IEEE Trans. Magn. MAG-18 (1982) 1107.

[3] W.H. Kraan and M.Th. Rekveldt, J. Magn. Magn. Mat. 5 (1977) 247.

[4] K. Hemmes, J.C. Lodder, M.Th. Rekveldt and W.H. Kraan, J. Phys. D17 (1984) L157.

[5] W.H. Kraan, M.Th. Rekveldt and J.J. van Loef, J. Magn. Magn. Mat. 21 (1980) 51.

[6] I. Privorotskii, Thermodynamic Theory of Domain Structures (J. Wiley \& Sons, New York/Toronto, 1976) ch. II

[7] A. Hubert, Proc. Intermag 85, St. Paul (1985), in press. 